\title{
EQUIVALENCE BETWEEN VARIOUS VERSIONS OF THE SELF-DUAL ACTION OF THE ASHTEKAR FORMALISM
}

\author{
J. A. Nieto ${ }^{1}$ \\ Facultad de Ciencias Físico-Matemáticas de la Universidad Autónoma \\ de Sinaloa, 80010, Culiacán Sinaloa, México
}

\begin{abstract}
Different aspects of the self-dual (anti-self-dual) action of the Ashtekar canonical formalism are discussed. In particular, we study the equivalences and differences between the various versions of such an action. Our analysis may be useful for the development of Ashtekar formalism in eight dimensions.
\end{abstract}

Pacs numbers: 04.60.-m, 04.65.+e, 11.15.-q, 11.30.Ly April, 2004

\footnotetext{
${ }^{1}$ nieto@uas.uasnet.mx
} 
Recently, Ashtekar and Lewandowski [1] reported a pedagogical presentation of loop quantum gravity. In particular, in its review article they consider the action

$$
S_{1}=\frac{1}{4} \int_{\mathcal{M}} \epsilon_{I J K L} e^{I} \wedge e^{J} \wedge \Omega^{K L}-\frac{1}{2 \gamma} \int_{\mathcal{M}} e^{I} \wedge e^{J} \wedge \Omega_{I J}
$$

as the starting point in the program of non-perturbative quantum gravity. Here, we closely follow the notation in Ref. [1]. $\mathcal{M}$ denotes a four dimensional spacetime manifold, $e^{I}$ are co-tetrads defined in $T_{x} \mathcal{M}$ for each $x \in \mathcal{M}, \epsilon_{I J K L}$ is a completely antisymmetric tensor compatible with the invariant metric $\eta_{I J}=\operatorname{diag}( \pm 1,1,1,1)$ of the group $S O(1,3)$ or $S O(4)$ and

$$
\Omega \equiv d \omega+\omega \wedge \omega
$$

The quantity $\gamma$ is a number called the Barebero-Immirzi parameter. If the signature of $\eta_{I J}$ is $0+4$ the interesting geometrical structure arises when $\gamma= \pm 1$, while if the signature of $\eta_{I J}$ is Lorenziana one has $\gamma= \pm i$. Just for simplicity, we shall focus in the case $\gamma=i$. Nevertheless, most of our computations also apply to the cases $\gamma=-i$ and $\gamma= \pm 1$.

The abstract notation in (1) is, in some sense, elegant but some times for computations is not so practical as a tensorial notation. For this reason, in order to clarify some aspects of the action (1) it becomes convenient to rewrite (1) in a tensorial notation. Since $e^{I}$ and $\Omega^{K l}$ are one-form and twoform respectively we have $e^{I}=e_{\mu}^{I} d x^{\mu}$ and $\Omega^{K L}=\frac{1}{2} \Omega_{\mu \nu}^{K L} d x^{\mu} \wedge d x^{\nu}$, where

$$
\Omega_{\mu \nu}^{K l}=\partial_{\mu} \omega_{\nu}^{K L}-\partial_{\nu} \omega_{\mu}^{K L}+\omega_{\mu}^{K S} \omega_{\nu S}^{L}-\omega_{\mu}^{L S} \omega_{\nu S}^{K} .
$$

Let write the action (1) in terms of $e_{\mu}^{I}$ and $\Omega_{\mu \nu}^{K L}$. One has

$$
S_{1}=\frac{1}{8} \int d^{4} x \epsilon^{\mu \nu \alpha \beta} \epsilon_{I J K L} e_{\mu}^{I} e_{\nu}^{J} \Omega_{\alpha \beta}^{K L}+\frac{i}{8} \int d^{4} x \epsilon^{\mu \nu \alpha \beta} \delta_{I J R S} e_{\mu}^{I} e_{\nu}^{J} \Omega_{\alpha \beta}^{R S} .
$$

Here, we used the notation $\delta_{I J R S}=\eta_{I R} \eta_{J S}-\eta_{I S} \eta_{J R}$ and the fact that

$$
\int d^{4} x \epsilon^{\mu \nu \alpha \beta}=\int_{\mathcal{M}} d x^{\mu} \wedge d x^{\nu} \wedge d x^{\alpha} \wedge d x^{\beta}
$$

Now, considering that

$$
\epsilon_{I J K L} \epsilon_{R S}^{K L}=-2 \delta_{I J R S}
$$


and by using the notation

$$
\Sigma_{\mu \nu}^{I J} \equiv e_{\mu}^{I} e_{\nu}^{J}-e_{\mu}^{J} e_{\nu}^{I}
$$

one sees that the action (4) can also be rewritten as

$$
S_{1}=\frac{1}{16} \int d^{4} x \epsilon^{\mu \nu \alpha \beta} \epsilon_{I J K L} \Sigma_{\mu \nu}^{I J} \Omega_{\alpha \beta}^{K L}-\frac{i}{32} \int d^{4} x \epsilon^{\mu \nu \alpha \beta} \epsilon_{I J K L} \epsilon_{R S}^{K L} \Sigma_{\mu \nu}^{I J} \Omega_{\alpha \beta}^{R S} .
$$

It is straightforward to see that this action is equivalent to

$$
S_{1}=\frac{1}{8} \int d^{4} x \epsilon^{\mu \nu \alpha \beta} \Sigma_{\mu \nu}^{I J+} \Omega_{\alpha \beta}^{K L} \epsilon_{I J K L}
$$

where

$$
{ }^{+} \Omega_{\alpha \beta}^{K L} \equiv \frac{1}{2}\left(\Omega_{\alpha \beta}^{K L}-\frac{i}{2} \epsilon_{R S}^{K L} \Omega_{\alpha \beta}^{R S}\right) .
$$

Thus, we have proved step by step that (1) is equivalent to (9).

It turns out that an alternative, but equivalent, way to write (9) is

$$
S_{1}=\int d^{4} x e e_{K}^{\alpha} e_{L}^{\beta+} \Omega_{\alpha \beta}^{K L} .
$$

Here, we used (7) and the property that $\epsilon^{\mu \nu \alpha \beta} e_{\mu}^{I} e_{\nu}^{J} \epsilon_{I J K L}=2 e \Sigma_{K L}^{\alpha \beta}$, with $e=\operatorname{det}\left(e_{\mu}^{I}\right)$. One recognizes in the expression (11) the action proposed by Jacobson and Smolin [2] and Samuel [3]. Therefore, the action (1) called in Ref. [1] the Holst action [4] is just the same as the one proposed by Jacobson-Smolin-Samuel for $\gamma=i$.

An important aspect of (11) or (9) is that it can be reduced to the real Einstein-Hilbert action

$$
S_{E H}=\frac{1}{2} \int d^{4} x e e_{K}^{\alpha} e_{L}^{\beta} \Omega_{\alpha \beta}^{K L}
$$

or

$$
S_{E H}=\frac{1}{16} \int d^{4} x \epsilon^{\mu \nu \alpha \beta} \Sigma_{\mu \nu}^{I J} \Omega_{\alpha \beta}^{K L} \epsilon_{I J K L}
$$

This result holds by the following reasons. First of all, observe that $+\Omega_{\alpha \beta}^{K L}$ is self-dual,

$$
{ }^{*+} \Omega_{\alpha \beta}^{K L} \equiv i^{+} \Omega_{\alpha \beta}^{K L} .
$$


Here, we used the definition ${ }^{*+} \Omega_{\alpha \beta}^{K L}=\frac{1}{2} \epsilon_{R S}^{K L+} \Omega_{\alpha \beta}^{R S}$. Therefore, ${ }^{+} \Omega_{\alpha \beta}^{K L}$ keeps only the self-dual part of the real curvature $\Omega_{\alpha \beta}^{K L}$. It turns out that, as Jacobson and Smolin emphasize [23], in four dimensions one has the algebra isomorphism $s o(1,3)=s u(2) \times s u(2)$ and consequently the self-dual curvature $+\Omega_{\alpha \beta}^{K L}$ can be combined with the anti-self-dual curvature

$$
-\Omega_{\alpha \beta}^{K L}=\frac{1}{2}\left(\Omega_{\alpha \beta}^{K L}+\frac{i}{2} \epsilon_{R S}^{K L} \Omega_{\alpha \beta}^{R S}\right)
$$

in the form

$$
\Omega_{\alpha \beta}^{K L}\left({ }^{+} \omega+{ }^{-} \omega\right)=\Omega_{\alpha \beta}^{K L}\left({ }^{+} \omega\right)+\Omega_{\alpha \beta}^{K L}\left({ }^{-} \omega\right) .
$$

Here, we used the fact that $\Omega_{\alpha \beta}^{K L}\left({ }^{ \pm} \omega\right)={ }^{ \pm} \Omega_{\alpha \beta}^{K L}$, where

$$
{ }^{ \pm} \omega_{\alpha}^{K L}=\frac{1}{2}\left(\omega_{\alpha}^{K L} \mp \frac{i}{2} \epsilon_{R S}^{K L} \omega_{\alpha}^{R S}\right) .
$$

Considering these results one finds that the equation of motion derived from the action (11) under variations with respect to ${ }^{+} \omega$ leads to the cyclic Bianchi identity for $\Omega_{\alpha \beta}^{K L}(e)$ and therefore the second term in (8), or (4), vanishes identically (see Ref. [2] for more details).

A variant of the action (9) is provided by the action

$$
S_{2}=\frac{1}{8} \int d^{4} x \epsilon^{\mu \nu \alpha \beta+} \Sigma_{\mu \nu}^{I J+} \Omega_{\alpha \beta}^{K L} \delta_{I J K L},
$$

where ${ }^{ \pm} \Sigma_{\mu \nu}^{I J}$ is the (anti-)self-dual part of $\Sigma_{\mu \nu}^{I J}$ :

$$
{ }^{ \pm} \Sigma_{\mu \nu}^{I J}=\frac{1}{2}\left(\Sigma_{\mu \nu}^{I J} \mp \frac{i}{2} \epsilon_{R S}^{K L+} \Sigma_{\mu \nu}^{R S}\right) .
$$

In abstract notation the action (18) becomes

$$
S_{2}=\int_{\mathcal{M}} \Sigma_{(+)}^{I J} \wedge \Omega_{I J}^{(+)}
$$

Here, $\Sigma_{(+)}^{I J}=\frac{1}{2}^{+} \Sigma_{\mu \nu}^{I J} d x^{\mu} \wedge d x^{\nu}$ and $\Omega_{I J}^{(+)}=\frac{1}{2}^{+} \Omega_{\alpha \beta}^{K L} d x^{\alpha} \wedge d x^{\beta}$. It turns out that the action (20), called $S_{(H)}$ in Ref. [1] (see expression (2.9) in Ref. [1]), also plays an essential role in the canonical quantization of gravity in four dimensions (see [1] and Refs. therein).

In this work, we shall show that the actions (9) and (18) (or equivalent (1) and (20)) can be considered as part of the action 


$$
S_{3}^{(+)}=\frac{1}{4} \int_{\mathcal{M}} \mathcal{F}^{(+) I J} \wedge \mathcal{F}^{(+) K L} \epsilon_{I J K L},
$$

which was proposed in Ref. [5] and generalized to the supersymmetric case in Ref. [6]. Here, the de Sitter curvature $\mathcal{F}^{I J}=\frac{1}{2} \mathcal{F}_{\mu \nu}^{I J} d x^{\mu} \wedge d x^{\nu}$ is defined as

$$
\mathcal{F}_{\mu \nu}^{I J}=\Omega_{\mu \nu}^{I J}+\Sigma_{\mu \nu}^{I J} .
$$

First, observe the action (21) can be rewritten as

$$
S_{3}=\frac{1}{16} \int d^{4} x \epsilon^{\mu \nu \alpha \beta+} \mathcal{F}_{\mu \nu}^{I J+} \mathcal{F}_{\alpha \beta}^{K L} \epsilon_{I J K L} .
$$

Let us now write ${ }^{+} \mathcal{F}_{\mu \nu}^{I J}$ in the form

$$
{ }^{+} \mathcal{F}_{\mu \nu}^{I J}=\frac{1}{2}^{+} B_{K L}^{I J} \mathcal{F}_{\mu \nu}^{K L},
$$

where

$$
{ }^{ \pm} B_{K L}^{I J}=\frac{1}{2}\left(\delta_{K L}^{I J} \mp i \epsilon_{K L}^{I J}\right) .
$$

By straightforward computation we find that the projector ${ }^{ \pm} B_{K L}^{I J}$ has the following interesting properties:

$$
\begin{gathered}
{ }^{ \pm} B_{M N}^{I J} \epsilon_{I J K L}= \pm 2 i^{ \pm} B_{M N K L}, \\
{ }^{ \pm} B_{M N}^{I J} \delta_{I J K L}=2^{ \pm} B_{M N K L}, \\
{ }^{ \pm} B_{M N}^{I J \pm} B_{R S}^{K L} \epsilon_{I J K L}= \pm 4 i^{ \pm} B_{M N R S}
\end{gathered}
$$

and

$$
{ }^{ \pm} B_{M N}^{I J \pm} B_{R S}^{K L} \delta_{I J K L}=4^{ \pm} B_{M N R S} .
$$

Moreover, (28) and (29) can be added to give

$$
{ }^{ \pm} B_{M N}^{I J \pm} B_{R S}^{K L \pm} B_{I J K L}=4^{ \pm} B_{M N R S} .
$$

Now, consider the alternative action 


$$
S_{4}=\frac{1}{16} \int d^{4} x \epsilon^{\mu \nu \alpha \beta+} \mathcal{F}_{\mu \nu}^{I J+} \mathcal{F}_{\alpha \beta}^{K L} \delta_{I J K L}
$$

By using the relations (28) and (29) one discovers that

$$
S_{3}=+i S_{4}
$$

Therefore, up to the complex numerical factor $i$ the actions $S_{3}$ and $S_{4}$ are equal. Thus, one can use either $S_{3}$ or $S_{4}$ to get the same gravitational information.

Let us now focus on the action $S_{4}$. Using (22) one sees that $S_{4}$ leads to

$$
\begin{gathered}
S_{4}=\frac{1}{16} \int d^{4} x \epsilon^{\mu \nu \alpha \beta+} \Omega_{\mu \nu}^{I J+} \Omega_{\alpha \beta}^{K L} \delta_{I J K L}+\frac{1}{8} \int d^{4} x \epsilon^{\mu \nu \alpha \beta+} \Sigma_{\mu \nu}^{I J+} \Omega_{\alpha \beta}^{K L} \delta_{I J K L} \\
+\frac{1}{16} \int d^{4} x \epsilon^{\mu \nu \alpha \beta+} \Sigma_{\mu \nu}^{I J+} \Sigma_{\alpha \beta}^{K L} \delta_{I J K L} .
\end{gathered}
$$

The first term in (33) corresponds to the complex sum of Euler and Prontrjagin topological invariants. The last term refers to the cosmological constant term. While the second term corresponds to the tensorial version of the action (20) (see action (18)). Therefore, we have proved that the action (18) is obtained from (31) when one dropps from $S_{4}$ the Euler and Prontrjagin topological invariants and the cosmological term. But, since $S_{4}$ is classically equivalent to the action $S_{3}$ this also proves that the action (18) or the action (20) are contained in the action $S_{3}$.

Using the relations (28) and (29) it can be shown that action $S_{2}$, given in $(18)$ (or $(20)$ ), is equivalent to

$$
S_{5}=\frac{1}{8} \int d^{4} x \epsilon^{\mu \nu \alpha \beta+} \Sigma_{\mu \nu}^{I J+} \Omega_{\alpha \beta}^{K L} \epsilon_{I J K L} .
$$

In fact, we find that $S_{5}=i S_{2}$. Moreover, using the relations (26)-(29) one finds that $S_{5}$ can be written in a variety of equivalent ways:

$$
\begin{gathered}
S_{6}=\frac{i}{8} \int d^{4} x \epsilon^{\mu \nu \alpha \beta} \Sigma_{\mu \nu}^{I J} \Omega_{\alpha \beta}^{K L+} B_{I J K L}, \\
S_{7}=\frac{i}{8} \int d^{4} x \epsilon^{\mu \nu \alpha \beta} \Sigma_{\mu \nu}^{I J+} \Omega_{\alpha \beta}^{K L+} B_{I J K L}, \\
S_{8}=\frac{i}{4} \int d^{4} x \epsilon^{\mu \nu \alpha \beta} \Sigma_{\mu \nu}^{I J+} \Omega_{\alpha \beta I J},
\end{gathered}
$$




$$
S_{9}=\frac{1}{8} \int d^{4} x \epsilon^{\mu \nu \alpha \beta} \Sigma_{\mu \nu}^{I J+} \Omega_{\alpha \beta}^{K L} \epsilon_{I J K L}
$$

In fact, we find that $S_{5}=S_{6}=S_{7}=S_{8}=S_{9}$. In particular one observes that $S_{1}=S_{9}$ and therefore one discovers that $S_{1}=i S_{2}$.

Just for completeness let us write the actions (34)-(38) in abstract notation:

$$
\begin{aligned}
S_{5}= & \frac{1}{2} \int_{\mathcal{M}} \Sigma_{(+)}^{I J} \wedge \Omega^{(+) K L} \epsilon_{I J K L}, \\
S_{6}= & \frac{i}{2} \int_{\mathcal{M}} \Sigma^{I J} \wedge \Omega^{K L+} B_{I J K L}, \\
S_{7}= & \frac{i}{2} \int_{\mathcal{M}} \Sigma^{I J} \wedge \Omega^{(+) K L+} B_{I J K L}, \\
& S_{8}=i \int_{\mathcal{M}} \Sigma^{I J} \wedge \Omega_{I J}^{(+)}, \\
S_{9}= & \frac{1}{2} \int_{\mathcal{M}} \Sigma^{I J} \wedge \Omega^{(+) K L} \epsilon_{I J K L},
\end{aligned}
$$

If instead of self-dual sector $+\mathcal{F}^{I J}$ one considers the anti-self-dual sector ${ }^{-} \mathcal{F}^{I J}$ of $\mathcal{F}^{I J}$ one obtains the analogue action to $S_{3}^{(+)}$, namely

$$
S_{3}^{(-)}=\frac{1}{4} \int_{\mathcal{M}} \mathcal{F}^{(-) I J} \wedge \mathcal{F}^{(-) K L} \epsilon_{I J K L}
$$

Following similar steps as in the case of $S_{3}^{(+)}$one may obtain from $S_{3}^{(-)}$all the corresponding equivalent actions $S_{5}, \ldots, S_{9}$ given in (39)-(43) but with the sign $(+)$ replaced by the sign $(-)$.

Summarizing, we have proved that the actions (1) and (20), reported in the review [1], are particular cases of the more general action $S_{3}^{(+)}$(or $S_{3}^{(-)}$). Specifically, (1) and (20) are obtained when one discards from $S_{3}^{(+)}$the Euler and Pontrjagin topological invariants and the cosmological constant term.

From (16) and (22) one sees that $\mathcal{F}^{I J}=\mathcal{F}^{(+) I J}+\mathcal{F}^{(-) I J}$ and consequently one finds

$$
\begin{aligned}
& S_{M M}=\frac{1}{4} \int_{\mathcal{M}} \mathcal{F}^{I J} \wedge \mathcal{F}^{K L} \epsilon_{I J K L}=\frac{1}{4} \int_{\mathcal{M}} \mathcal{F}^{(+) I J} \wedge \mathcal{F}^{(+) K L} \epsilon_{I J K L} \\
& +\frac{1}{4} \int_{\mathcal{M}} \mathcal{F}^{(-) I J} \wedge \mathcal{F}^{(-) K L} \epsilon_{I J K L}+\frac{1}{2} \int_{\mathcal{M}} \mathcal{F}^{(+) I J} \wedge \mathcal{F}^{(-) K L} \epsilon_{I J K L} .
\end{aligned}
$$


One recognizes the action $S_{M M}$ as the usual MacDowell-Mansouri action [7] (see also Ref. [8]) which can be derived by breaking the De Sitter gauge group $S O(1,4)$ (or anti-De Sitter $S O(2,3)$ ) to the smaller group $S O(1,3)$. In fact, in the MacDowell-Mansouri theory the connection $\omega^{I J}$ associated with the group $S O(1,3)$ and the tetrad $e^{I}$ arise after breaking the original De Sitter connection $\omega^{\hat{I}} \hat{J}$ where $\hat{I}$ and $\hat{J}$ are group indices of the De Sitter group $S O(1,4)$ (or anti-De Sitter group $S O(2,3)$ ). Consequently, $S_{3}^{(+)}$(or $\left.S_{3}^{(-)}\right)$carries the self-dual sector of the De Sitter gauge group property of $S_{M M}$.

On purpose to study $S$-duality for gravity García-Compeán et al [8] modified the action (45) in the form

$$
\mathcal{S}_{3}=\frac{{ }^{+} \tau}{4} \int_{\mathcal{M}} \mathcal{F}^{(+) I J} \wedge \mathcal{F}^{(+) K L} \epsilon_{I J K L}+\frac{{ }^{-} \tau}{4} \int_{\mathcal{M}} \mathcal{F}^{(-) I J} \wedge \mathcal{F}^{(-) K L} \epsilon_{I J K L}
$$

where ${ }^{+} \tau$ and ${ }^{-} \tau$ are constant coupling parameters. Indeed, the action (46) is the bosonic sector a more general supersymmetric action (see Ref. [9] for details).

Other generalization of $S_{3}^{(+)}$seems to lack of the attractive gauge properties contained in MacDowell-Mansouri procedure. In particular, by combining duality in the spacetime indices and the group indices Soo [10] extended the action $S_{3}^{(+)}$to a positive definite action. Montesinos [11] added to (1) the Euler and Pontrjagin topological invariants with constant factor parameters. Obukhov and Hehl [12] analized also a number of possibilities by combining dualities in the spacetime indices and the group indices. However, since a duality associated with the spacetime indices requires a metric these extensions of $S_{3}^{(+)}$can not be considered as genuine gauge theories in the sense of the MacDowell-Mansouri theory.

Besides the important gauge properties of the action $S_{3}^{(+)}$one may become interested in this action because of its closeness to topological features. In fact, as has been pointed out [5], if instead of the fully antisymmetric symbol $\epsilon_{I J K L}$ one uses the Killing metric associated with the De Sitter group $S O(1,4)$ (or $S O(2,3)$ ) the action $S_{3}^{(+)}$becomes the second Chern class. This means that the action $S_{3}^{(+)}$which leads to the Ashtekar formalism is closely related to the Chern-Simons action. Thus, a canonical quantization of $S_{3}^{(+)}$may lead to the intriguing result that an 'almost' a Chern-Simons action, as is the case of $S_{3}^{(+)}$, leads to physical states which are exponential of the Chern-Simons 
action which are predicted by the quantum general relativity of the canonical Ashtekar formalism (see [13]-[14]).

Finally, the present work may be useful in the recent proposal [15] of extending the Ashtekar formalism to eight dimensions. It turns out that, by using an octonionic structure [16]-[17], in the Ref. [15] the analogue of the action $S_{3}^{(+)}$was proposed in a spacetime of signature $1+7$. Thus, a revisited analysis of the action $S_{3}^{(+)}$, as presented in this work, seems to be a necessary step for further development in such an eight dimensional program.

\section{References}

[1] A. Ashtekar and J. Lewandowski, Class. Quant. Grav. 21, R53 (2004); gr-qc/0404018.

[2] T. Jacobson and L. Smolin, Class. Quant. Grav. 5, 583 (1988).

[3] J. Samuel, Pramana J. Phys. 28, L429 (1987).

[4] S. Holst, Phys. Rev. D 53, 5966 (1996); gr-qc/9511026.

[5] J. A. Nieto, O. Obregón and J. Socorro, Phys. Rev. D 50, R3583 (1994); gr-qc/9402029.

[6] J. A. Nieto, J. Socorro and O. Obregón, Phys. Rev. Lett. 76, 3482 (1996).

[7] S. W. MacDowell and F. Mansouri, Phys. Rev. Lett. 38, 793 (1977); F. Mansouri, Phys. Rev. D 16, 2456 (1977).

[8] P. G. O. Freund, Introduction to Supersymmetry (C. U. P. 1986).

[9] H. García-Compeán, J. A. Nieto, O. Obregón and C. Ramírez, Phys. Rev. D 59, 124003 (1999); hep-th/9812175.

[10] C. Soo, Phys. Rev. D 52, 3484 (1995); gr-qc/9504042.

[11] M. Montesinos, Class. Quant. Grav. 18, 1847 (2001); gr-qc/0104068.

[12] Y. N. Obukhov and F. W. Hehl, Acta Phys. Polon. B 27, 2685 (1996); gr-qc/9602014. 
[13] H. Kodama, Phys. Rev. D 42, 2548 (1990).

[14] B. Brugmann, R. Gambini and J. Pullin, Nucl. Phys. B 385, 587 (1992); hep-th/9202018.

[15] J. A. Nieto, Class. Quant. Grav. 22, 947 (2005); hep-th/0410260.

[16] J. C. Baez, Bull. Amer. Math. Soc. 39, 145 (2002).

[17] S. Okubo, Introduction to Octonion and Other Non-Associative Algebras (Cambridge University Press, 1995). 